\title{
Potential Role of Peptidylarginine Deiminase Enzymes and Protein Citrullination in Cancer Pathogenesis
}

\author{
Sunish Mohanan, ${ }^{1}$ Brian D. Cherrington, ${ }^{2}$ Sachi Horibata, ${ }^{1}$ John L. McElwee, ${ }^{1}$ \\ Paul R. Thompson, ${ }^{3}$ and Scott A. Coonrod ${ }^{1}$ \\ ${ }^{1}$ Baker Institute for Animal Health and Department of Biomedical Sciences, Cornell University, Hungerford Hill Road, Ithaca, \\ NY 14853-6401, USA \\ ${ }^{2}$ Department of Zoology and Physiology, University of Wyoming, Laramie, WY 82071, USA \\ ${ }^{3}$ Department of Chemistry, The Scripps Research Institute, FL 33458, USA
}

Correspondence should be addressed to Scott A. Coonrod, sac269@cornell.edu

Received 29 May 2012; Revised 31 July 2012; Accepted 9 August 2012

Academic Editor: Rolf J. Craven

Copyright () 2012 Sunish Mohanan et al. This is an open access article distributed under the Creative Commons Attribution License, which permits unrestricted use, distribution, and reproduction in any medium, provided the original work is properly cited.

The peptidylarginine deiminases (PADs) are a family of posttranslational modification enzymes that catalyze the conversion of positively charged protein-bound arginine and methylarginine residues to the uncharged, nonstandard amino acid citrulline. This enzymatic activity is referred to as citrullination or, alternatively, deimination. Citrullination can significantly affect biochemical pathways by altering the structure and function of target proteins. Five mammalian PAD family members (PADs 1-4 and 6) have been described and show tissue-specific distribution. Recent reviews on PADs have focused on their role in autoimmune diseases. Here, we will discuss the potential role of PADs in tumor progression and tumor-associated inflammation. In the context of cancer, increasing clinical evidence suggests that PAD4 (and possibly PAD2) has important roles in tumor progression. The link between PADs and cancer is strengthened by recent findings showing that treatment of cell lines and mice with PAD inhibitors significantly suppresses tumor growth and, interestingly, inflammatory symptoms. At the molecular level, transcription factors, coregulators, and histones are functional targets for citrullination by PADs, and citrullination of these targets can affect gene expression in multiple tumor cell lines. Next generation isozyme-specific PAD inhibitors may have therapeutic potential to regulate both the inflammatory tumor microenvironment and tumor cell growth.

\section{Introduction}

PAD-mediated citrullination can alter the tertiary structure of target substrates and/or alter protein-protein interactions; thus affecting various cellular processes $[1,2]$ (Figure 1). Recently, protein citrullination has garnered increased attention due to its role in the pathogenesis of various inflammatory conditions such as rheumatoid arthritis (RA), multiple sclerosis, psoriasis, chronic obstructive pulmonary disease (COPD), neurodegenerative diseases and, also, due to its emerging role in various human and animal cancers [3-7]. In this paper, we will first briefly discuss the tissue-specificity and hormonal regulation of the five PAD isoforms and then focus on the potential role of this enzyme family in carcinogenesis, tumor progression, and inflammation.

\section{Tissue Expression Patterns and Substrate Specificity of PAD Family Members}

PADs are $\mathrm{Ca}^{2+}$-dependent enzymes and there are five different isozymes in mammals, namely, PAD1, 2, 3, 4, and $6[8,9]$. The PAD genes likely arose by duplication of the ancestral homologue, $P A D 2$, and are localized to a wellorganized gene cluster at 1p36.13 in humans. Interestingly, this locus is also predicted to contain a novel, yet to be defined, tumor suppressor protein [10]. PAD enzymes are highly homologous, with $\sim 50-60$ percent sequence identity at the amino acid level. Although there is some overlap with respect to target proteins, each family member also appears to target a unique set of cellular proteins as well $[11,12]$. Additionally, as described below, each family member also 

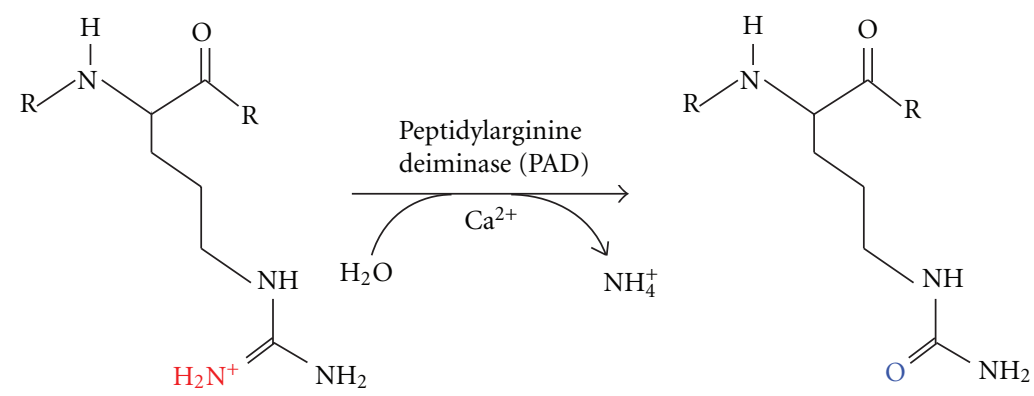

FIGURE 1: Peptidylarginine deiminase (PAD) enzymes catalyze the conversion of protein arginine residues to citrulline.

exhibits a tissue-selective distribution pattern. We note here that, in this paper, we will be using the more commonly utilized gene synonym, $P A D$, while the approved HUGO Gene Nomenclature Committee gene names for the family members are actually PADI1, PADI2, PADI3, PADI4, and PADI6.

PAD1 appears to primarily be expressed in the epidermis and uterus. In the epidermis, PAD1 citrullinated cytokeratin and this modification is important for modulating the cornification of the epidermis and maintaining the barrier function of superficial keratinized epidermal cell layers. The loss of charge following citrullination of cytokeratin causes disassembly of the cytokeratin-filaggrin complex and proteolytic degradation of these targets.

PAD2 is expressed in multiple organs, including the brain, female reproductive tissues, skeletal muscle, and cells of the hematopoietic lineage. In the brain, a major target for $\mathrm{PAD} 2$ is myelin basic protein (MBP), a constituent of the myelin sheath $[13,14]$. Citrullination of MBP by PAD2 likely plays a key role in the pathogenesis of neurodegenerative disease [15]. PAD2 can also citrullinate the intermediate filament vimentin in macrophages [16], leading to cytoskeletal disintegration and eventually apoptosis. In female reproductive tissues, PAD2 levels appear to be regulated by hormones, predominantly estrogen, and also possibly by epidermal growth factor [17]. PAD2 has also been found to be expressed in human mammary gland epithelial cells, with a fraction of PAD2 in these cells localizing to the nucleus and binding directly to chromatin [18]. In canine mammary tissue, histone citrullination levels closely correlate with the expression of PAD2 across the estrous cycle, suggesting that PAD2 may target histones for citrullination in this tissue [17]. In support of this prediction, a recent study has found that PAD2-catalyzed citrullination of histone $\mathrm{H} 3$ arginine 26 can regulate estrogen receptor $\alpha$ target gene activity [19].

PAD3 expression is highly restricted to the hair follicle and epithelium and a major target for PAD3 is trichohyaline. Additionally PAD3 can also citrullinate filaggrin leading to altered epidermal homeostasis and loss of barrier function [20].

PAD4 is expressed in hematopoietic progenitor cells, immune cells such as granulocytes, monocytes and macrophages, natural killer cells, and carcinoma cells originating from lung, esophagus, breast, and ovary $[5,21]$. PAD4 is often localized to the nucleus and is the only
PAD family member with a canonical nuclear localization sequence [22]. Antithrombin has been found to be an extracellular PAD4 substrate [23] and citrullination of this target suppresses the ability of antithrombin to inhibit thrombin [24]. Increased thrombin activity is considered to be a hallmark of cancer by promoting angiogenesis, increased tumor growth, and distant metastasis. Interestingly, citrullinated antithrombin levels are elevated in serum samples from patients with malignant cancers, thus raising the possibility that PADs may affect tumor progression via citrullination of antithrombin [25].

PAD4 also appears to function as a transcriptional coregulator for a range of factors such as p53, ELK1, p300, p21, CIP1, nucleophosmin, and ING4 [26-30]. While the mechanism by which PAD4 regulates target gene activity is not entirely clear, Edman degradation and analysis using sitespecific anticitrullinated histone antibodies has found that PAD4 can target the N-terminal tails of histones H2A, H3, and $\mathrm{H} 4$ for citrullination. More specifically, PAD4 has been found to directly citrullinate histone $\mathrm{H} 4$ and $\mathrm{H} 2 \mathrm{~A}$ at arginine 3 , and histone $\mathrm{H} 3$ at arginines' 2, 8, 17, and 26. Histone tail citrullination has been found to promote chromatin decondensation in vitro and in vivo $[31,32]$. Thus, it seems likely that the gene regulatory role of PAD4 is mediated by its initial recruitment to target promoters by the relevant transcription factor, followed by subsequent deimination of specific residues in the $\mathrm{N}$-terminal histone tails, leading to local changes in chromatin architecture and modulation of target gene expression (Figure 2). PAD4 is both a corepressor and coactivator of gene transcription and also appears to contribute to epigenetic cross-talk [33] during DNA damage by acting in concert with histone deacetylase 2 (HDAC2) to regulate p53 target gene activity [34]. Following DNA damage, PAD4 and HDAC2 separate from the p53-target gene promoters such as $\mathrm{p} 21, \mathrm{GADD} 45$, and PUMA, resulting in an increased incidence of histone Lys acetylation and Arg methylation at these sites.

PAD6 is a maternal effect gene that is specifically expressed in oocytes and preimplantation embryos and is essential for embryonic development beyond the 2-cell stage [35]. To date, there has been little evidence that this ovarian PAD isozyme is involved in cancer. Interestingly, however, a genome-wide SNP association in Icelanders showed a significant correlation between cutaneous-basal cell carcinoma risk and mutations in the PAD4/PAD6 locus 


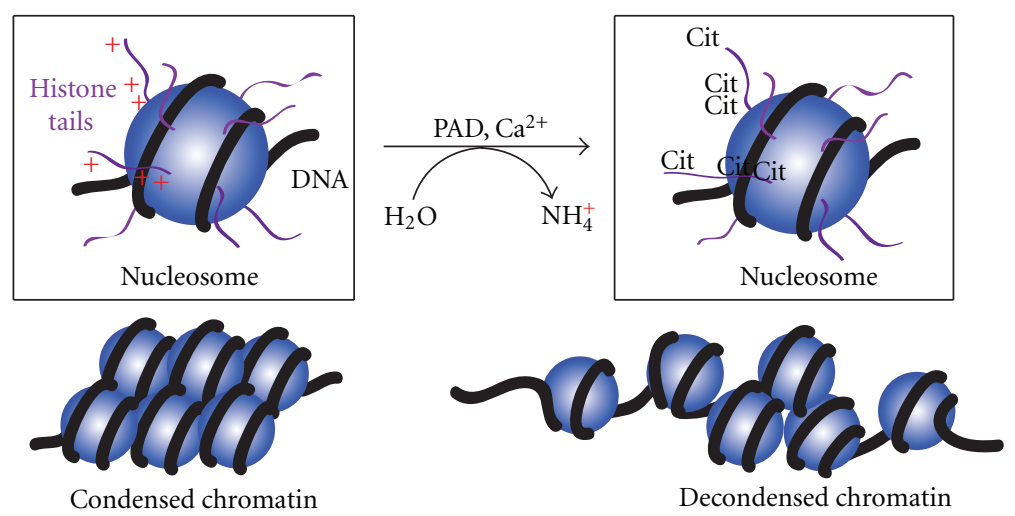

Figure 2: PAD-mediated histone tail citrullination leads to chromatin decondensation.

at 1 p36 [36]. The importance of this PAD family member in developmental biology is reviewed elsewhere $[37,38]$ and will not be discussed further in this paper.

\section{Hormonal Regulation of PADs}

Early PAD biological studies focused mainly on the expression patterns of the different family members in reproductive tissues and also on their catalytic activity at these sites. Outcomes from these studies showed that anterior pituitarylocalized rat lactotroph cells express PAD2 in a sexually dimorphic fashion, with expression confined to the female. Additional studies showed that PADs 1, 2, and 4 are present in uterine tissue and that protein citrullination is extensive in the uterine epithelium in an estrus cycle-dependent manner $[39,40]$. More recently, studies have found that mouse, dog, and human mammary glands express both PAD2 and 4 in luminal epithelial cells [17].

3.1. Pituitary Gland and Uterus. In the female rat pituitary, PAD enzymatic activity is highest during proestrus and estrus when serum estrogen levels are at their peak $[39,41]$. Ovariectomy of rats suppressed PAD activity in pituitary lysates, but activity could be restored by injection of exogenous estrogen. Furthermore, treatment of the pituitaryderived MtT/S cell line with estrogen results in a dosedependent increase in PAD expression and activity [42]. Together, these observations suggest that estrogen directly influences PAD expression and activity in the pituitary. Given that estrogen primes lactotrophs for prolactin biosynthesis, PAD enzymatic activity may play an important role in this normal physiological process and may also promote pituitary neoplastic growth.

Our examination of Massively Parallel Signature Sequencing (MPSS) transcriptome data (GEO Profiles GDS868) indicates that PADs 1, 2, and 4 expression levels are highest in the mouse uterus as compared to the 50 other tissues examined [43]. Furthermore, analysis of cDNA microarray data from Hewitt et al. shows that PAD1, 2, and
4 mRNA appear to be estrogen regulated [44]. In the uterus, the distribution of these isozymes appears to be primarily limited to glandular and luminal epithelial cells and also displays an estrous cycle-dependent regulation pattern, with the highest expression of these family members occurring during estrus $[45,46]$. Similar to the pituitary, PAD2 and PAD4 expression and activity in the uterus is lost following ovariectomy, but can be restored by injection of exogenous estrogen, indicative of estrogen regulation [45, 46]. Given the known mitogenic properties of estrogen in female cancers, it is possible that estrogen-induced upregulation of PAD expression and activity in the uterus may promote neoplastic growth in this tissue. However, a potential role for PADs in pituitary and uterine tumor progression has yet to be investigated.

3.2. Mammary Gland. Protein and mRNA expression studies in canine mammary glands collected at various stages of the estrous cycle show that PAD2 expression initiates during estrus, with mRNA and protein levels peaking during diestrus. In the mouse mammary gland PAD2 and 4 expression is highest in luminal epithelial cell populations during the estrus $[17,46]$. This species-specific difference in expression levels may reflect differences in estrous cycle stage lengths and hormone levels between species. Ovariectomy in mice also results in loss of PAD2 and 4 expression in the mammary gland, thus further corroborating the role of estrogen in PAD expression in female reproductive tissues. Molecular studies show that treatment of MCF7 cells with estrogen rapidly induced the upregulation of PAD4 and the involvement of estrogen in PAD4 expression in mammary epithelial cells appears to be mediated by two upstream estrogen response elements (designated ERE - 125 and -126) which are bound by $\operatorname{ER} \alpha$ following estrogen treatment. Furthermore, this study showed that the ER $\alpha$ dependent increase in PAD4 expression can also be mediated by estrogen signaling to the PAD4 proximal promoter via cross-talk with the AP-1, Sp-1, and NF-Y transcription factors [47]. 
DAPI

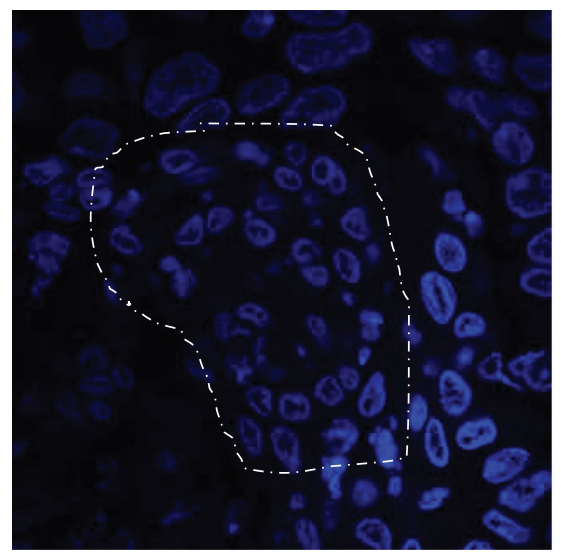

H4 Cit 3

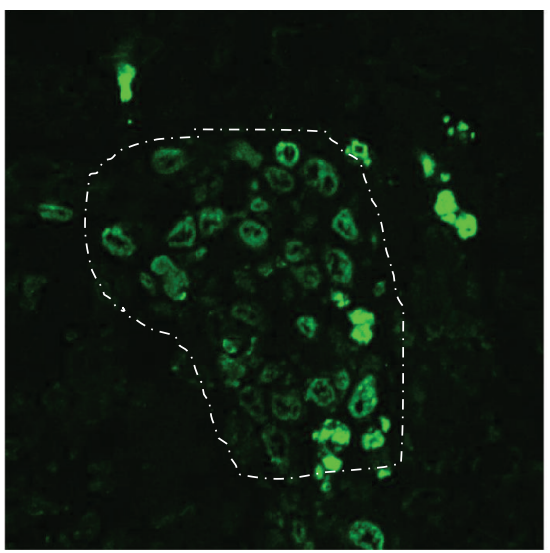

Merge

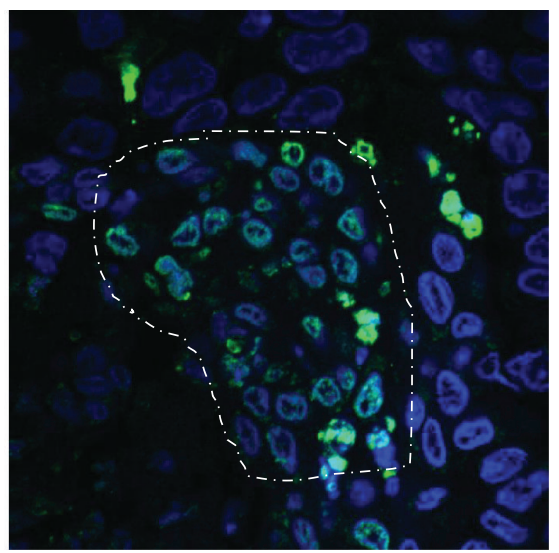

FIGURE 3: H4cit3 immunostaining of DCIS xenograft sections. Dotted line demarcates the area adjacent to the central necrotic core of the comedo-DCIS lesion. (Magnification 400x).

\section{PAD4-Mediated Histone Tail Citrullination: An Emerging Role for PADs in Gene Regulation and Cancer}

PAD4 has also been found to regulate estrogen receptor target gene activity following estrogen stimulation via histone tail citrullination [48]. Given the role of estrogen as a mitogen in cancer cells, this observation provides a clear potential link between PAD activity and cancer growth. In addition to estrogen, another mitogen, EGF, has been shown to utilize PAD4 as a cofactor to activate target gene activity. Zhang et al. documented that treatment of MCF-7 cells with EGF leads to PAD4-mediated citrullination of the ELK1 oncogene. This citrullination event then facilitated subsequent phosphorylation of ELK1 by ERK1/2, which, in turn, promoted histone acetylation and subsequent activation of a range of targets including the immediate early gene, c-fos [30].

PAD4 has also been found to interact with the major tumor suppressor, p53, and affect the expression of p53 target genes such as p21, OKL38, CIP1, and WAF1 [2630]. Interestingly, a recent study also found that citrullination levels at histone $\mathrm{H} 4$ arginine 3 (H4R3) are inversely correlated with p53 protein expression and with tumor size in nonsmall cell lung cancer tissues [32]. The authors also demonstrated that the p53-PAD4 pathway leads to citrullination of H4R3 and Lamin C in response to DNA damage and nuclear fragmentation. They also found that PAD4-mediated H4R3 citrullination appears to lead to localized chromatin decondensation around sites of DNA damage, thus facilitating p53-mediated cell death. In vivo studies then demonstrated that even though PAD4-null mice were grossly normal with regard to organ morphologies, they appeared resistant to apoptotic stimuli and also showed a consistent reduction in cleaved caspase- 3 expression. The authors conclude that the histone $\mathrm{H} 4$ citrulline 3 (H4Cit3) modification may form a novel "apoptotic code" which could potentially be used to detect a range of damaged cells, including tumor cells, following treatment of patients with cancer therapies. It is interesting to note, however, that the PAD inhibitor Cl-amidine increases p53 levels both in cell culture and in inflammatory cells isolated from mice treated with this compound $[29,49]$. Reconciling these two disparate observations requires further study. In support of a role for the H4Cit3 modification in marking apoptotic tumor cells, recent immunofluorescence studies in our lab found that citrullination at H4R3 was very robust within the nucleus of epithelial cells undergoing morphological changes associated with various stages of apoptosis in comedoDCIS xenograft sections (Figure 3 ). These xenografts were generated from MCF10DCIS cell line which belongs to the group of MCF10AT tumor progression series of cell lines $[50,51]$.

From a more clinical perspective, recent immunohistochemical and western blot studies have found that PAD4 appears to be overexpressed in several types of invasive carcinomas $[5,52,53]$. Outcomes from these studies found that PAD4 expression and, frequently activity, was elevated in neoplastic cells from breast carcinomas, lung adenocarcinomas, hepatocellular carcinomas, esophageal carcinomas with squamous differentiation, colorectal adenocarcinomas, renal carcinomas, ovarian adenocarcinomas, uterine carcinomas, uterine adenocarcinomas, and bladder carcinomas [52]. However, PAD4 expression was absent or minimal in the following tumors: benign gastric and uterine leiomyomas, hyperplastic conditions of endometrium, cervical polyps, teratomas, hydatidiform moles, hemangiomas, lymphatic proliferative conditions, schwannomas, and neurofibromas [5]. Consistent with the PAD4 protein expression pattern in carcinomas, the same patients also had elevated serum PAD4 activity and citrullinated antithrombin levels. Taken together, these observations support the potential use of this enzyme as a clinical prognostic biomarker. 
Normal

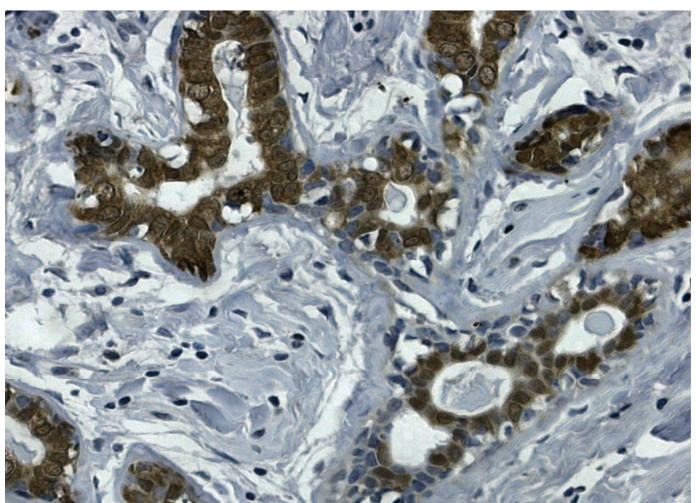

(a)

Invasive-low intensity staining

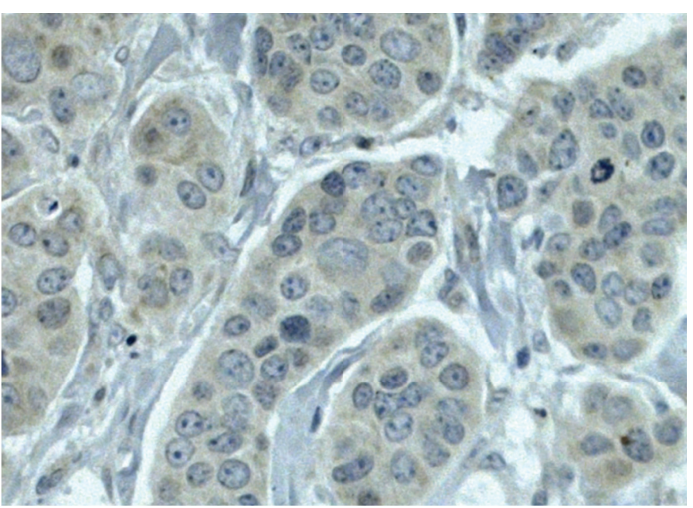

(c)
DCIS

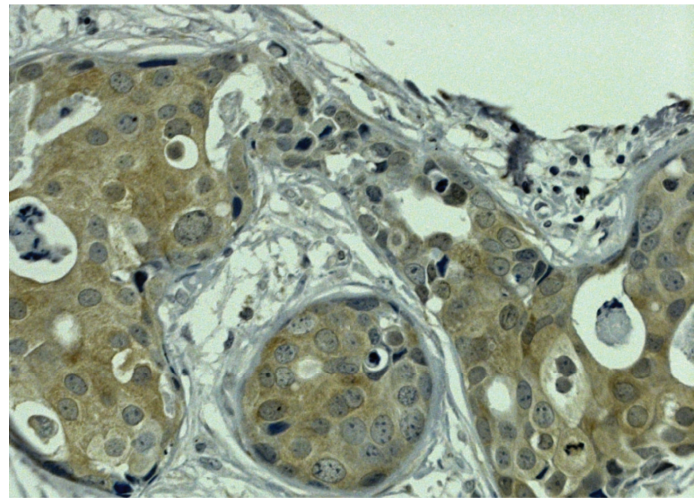

(b)

Invasive-high intensity staining

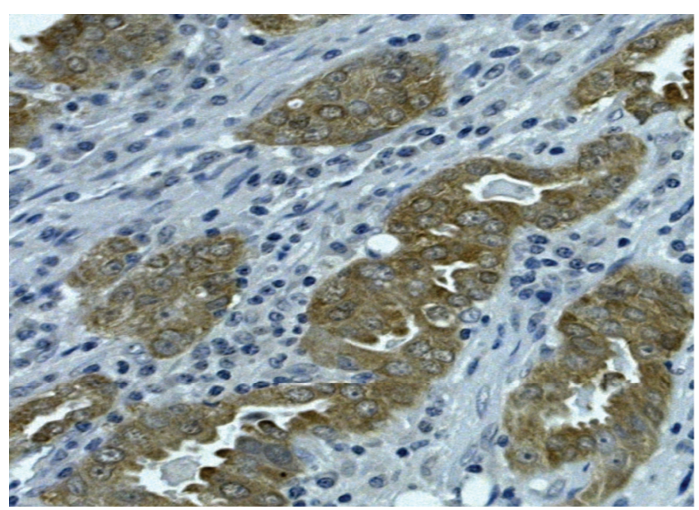

(d)

FIGURE 4: PAD2 IHC staining of the normal human mammary gland, a DCIS lesion, and invasive carcinomas. Nuclear staining intensity is reduced in most invasive tumors while a subset of these tumors retains strong PAD2 staining. (Magnification 200x).

DCIS

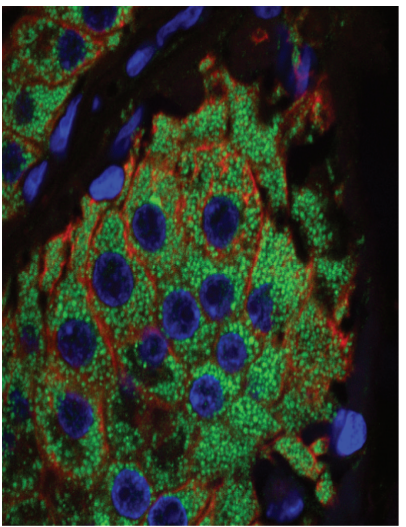

Invasive

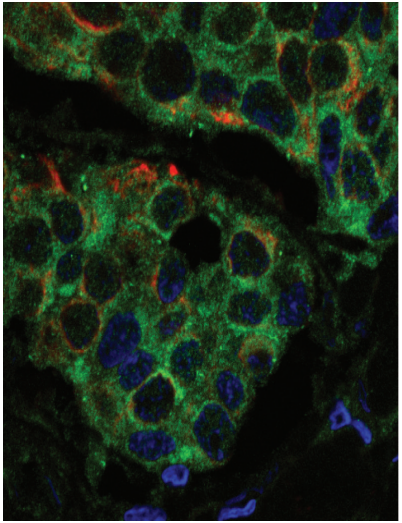

Metastatic

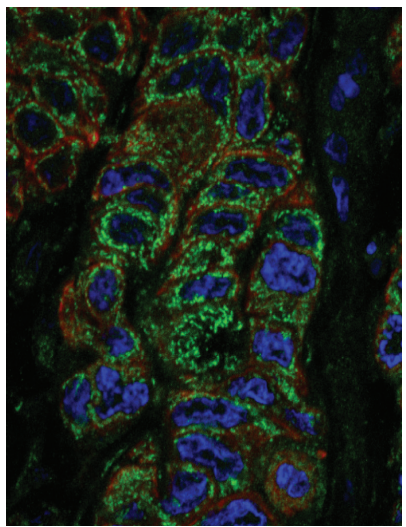

FIGURE 5: PAD2 (green) is expressed in luminal epithelial cells of preinvasive, invasive, and metastatic human mammary tumors. Cytokeratin (red) staining differentiates luminal epithelial type from myoepithelial cells. (Magnification 400x). 


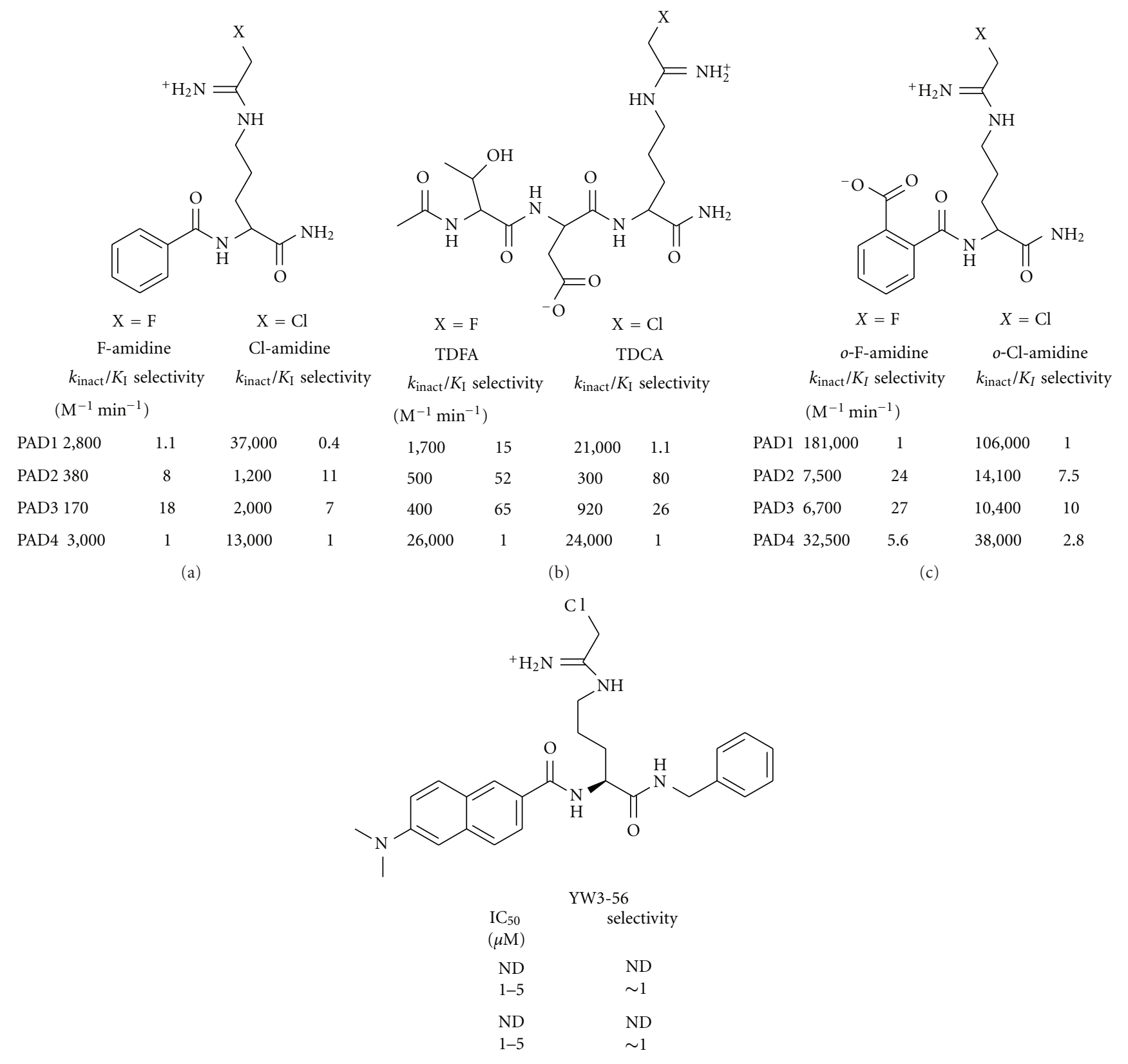

(d)

FIGURE 6: PAD inhibitors and their selectivity (TDFA-Threonine-aspartate-F-amidine; TDCA-Threonine-aspartate-Cl-amidine).

\section{PAD2 and Cancer Pathogenesis}

Given the volume of recent literature linking PAD4 with gene regulation in cancer cells, a role for this family member in tumor progression seems likely. Comparative studies evaluating PAD2 expression in mammary carcinomas from humans, dogs, and cats show that the nuclear localization of PAD2 may also prove to be associated with tumor progression. Normal human, canine, and feline mammary tissue shows strong nuclear and cytoplasmic PAD2 staining, but as tumors progress there is a general reduction in the nuclear localization of PAD2 (Figures 4 and 5) [54]. In the mammary gland, PAD2 expression is specific to cytokeratin positive luminal type epithelial cells (Figure 5 ). Thus, the loss of nuclear PAD2 may result in alterations in gene expression that lead to neoplastic transformation. It is interesting to note that a subset of invasive breast carcinomas tend to retain strong cytoplasmic and nuclear expression of PAD2. Further characterization of this subclass of tumors with regard to function of PAD2 is warranted as isozyme specific PAD inhibitors may be of use in combinatorial therapies to treat such tumor types.

Given these new findings, and PAD4's documented role in cancer biology, it will be interesting to determine whether 


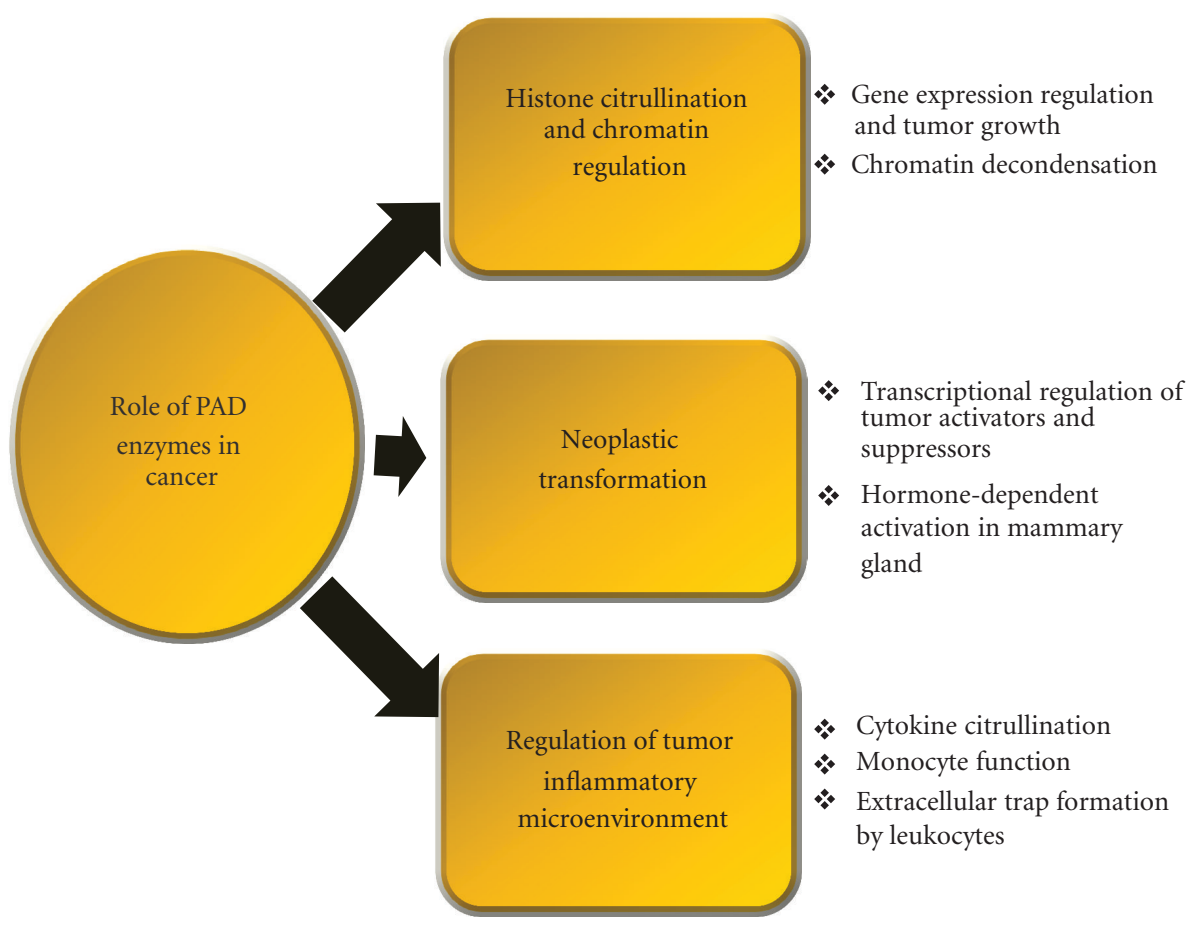

Figure 7: Potential role of PAD enzymes in cancer pathogenesis.

PAD2 and PAD4 may function in a synergistic manner to promote tumor progression. The observation that both PAD2 and PAD4 expression appears to be regulated at some level by estrogen, suggests that these two PADs may work together to mediate the estrogen response. The observations that PAD2 expression in mammary epithelial cells is induced by EGF [17], and that PAD4 regulates EGF-induced ELK1 target gene activation, suggests that PAD2 and PAD4 may also cooperate to mediate EGFR signaling [30].

\section{PAD Inhibitors Block Cancer Progression}

In further support of a role for PADs in tumor growth, several recent reports have also shown that treatment of cancer cell lines with PAD inhibitors decreases cancer cell viability without affecting the growth of normal cells [55]. Cl-amidine [56], and the related PAD4 inhibitor, F-amidine [57], display low micromolar cytotoxicity towards various tumor cell lines such as U2OS cells, HL-60, HT-29, and MCF-7 [29, 55, 58]. These compounds also can induce the differentiation of HL-60 cells, a leukemic cell line, making these cells more susceptible to drug treatments [55]. Clamidine can also act synergistically with the anticancer drug doxorubicin, thus enhancing the efficiency of cell death following a simultaneous treatment with these two compounds. In tumor, cell lines such as MCF-7 cells, Cl-amidine also regulates the expression of the tumor suppressor protein OKL38 in a p53-dependent manner by decreasing histone citrullination at the OKL38 promoter $[28,29]$.

A recent study, using a $\mathrm{Cl}$-amidine derivative with increased cell permeability, YW3-56 (Figure 6), found that this drug significantly suppressed cancer cell growth and also reduced tumor size in mouse xenograft models of sarcoma [59]. Furthermore, this compound affected the expression of genes related to cell proliferation and cell death and was also found to regulate macro-autophagy in cancer cells. Mechanistically, the authors discovered that the drug likely targeted factors within the mTORC1 pathway for inhibition. These studies, as well as the demonstration that PAD inhibitors are well tolerated in multiple different mouse disease models $[49,60]$, underscores the potential of PAD inhibitors as novel epigenetic anticancer drugs. Given that Cl-amidine, F-amidine, and YW3-56 display limited selectivity (Figure 6), it is unclear whether the inhibition of one or more PADs is required for the in vivo effects of these compounds. The development of new, more selective compounds, such as the PAD4 selective inhibitor TDFA and the PAD1 selective inhibitor o-F-amidine (Figure 6), will undoubtedly prove to be useful for sorting this issue out.

\section{PAD-Mediated Citrullination: Linking Inflammation with Cancer Progression?}

7.1. Role of Inflammation in Cancer Progression. Chronic inflammation is involved in the progression and recurrence of many types of cancer, including breast cancer. Epidemiological studies have documented that high levels of circulating acute phase inflammation-associated proteins at 3 years after-treatment are associated with an elevated risk for subsequent tumor recurrence and mortality in women [61]. Several studies have also demonstrated direct links between circulating inflammatory markers and progression 
to metastatic breast cancer [62-66]. Additionally, proinflammatory cytokines are well known for promoting tumor growth and facilitating metastasis by altering the tumor cell phenotype and by regulating stromal cells (endothelial cells, tumor-associated macrophages, and fibroblasts) within the tumor microenvironment. Furthermore, infiltrating immune cells within the tumor itself can promote tumorigenesis [67]. These findings suggest that the mechanisms by which inflammation in the tumor microenvironment drives metastasis is both intimately linked and fundamentally different to the primary mechanisms driving carcinogenesis [68].

7.2. Role of PADs in Inflammation. As noted earlier, numerous studies have documented increased protein citrullination within inflamed tissues from patients with autoimmune diseases such as rheumatoid arthritis and colitis [3, 4, 69]. More recently, these inflammatory symptoms have been shown to be suppressed by the PAD inhibitor, Cl-amidine, in mouse models of colitis and RA [49]. Another emerging link between PADs and inflammation is the newly defined role for PAD4 in catalyzing histone hypercitrullination during Neutrophil Extracellular Trap (NET) formation in inflamed tissues. A number of recent reports have shown that, following activation, peripheral blood neutrophils form a highly decondensed chromatin structure that both captures and kills invading pathogens [70-72]. Mechanistically, PAD4 was found to catalyze this dramatic chromatin decondensation event via histone tail hypercitrullination [73]. Analysis of this process at the ultrastructural level by electron microscopy showed that, following PAD4 activation in HL60 granulocytes, these cells show a dramatic and rapid conversion of multilobular heterochromatic nuclei to a more round euchromatic nuclear architecture, suggesting a direct role for PADs in heterochromatic-euchromatic interchange [31]. These new findings indicate that PAD4 can mediate chromatin structure change both at the local and genomewide level.

Citrullination of vimentin is correlated with the proliferation of fibroblast-like synoviocytes (following isolation from patients with rheumatoid arthritis) and also stimulates TNF- $\alpha$ and IL-1 production in these cells [74]. Given the links between vimentin citrullination, inflammation, and cell proliferation, and given how important cytoskeletal integrity is for cell motility, we hypothesize that citrullination of vimentin by PAD enzymes may also affect tumor cell migration and promote an inflammatory microenvironment. Additionally, given that PAD2 can regulate cytokine signaling in macrophages via citrullination of IKK $\gamma$ (thus suppressing NF-kB activity) [75], we predict that PAD-mediated regulation of macrophage activity could also potentially affect cross-talk between tumor-associated macrophages and cancer cells.

While PAD activity has primarily been found to regulate autoimmune-mediated inflammatory events, recent studies suggest that PAD-mediated citrullination is also elevated in a variety of inflammatory states which lack a strong autoimmune component, such as COPD and myositis [7678]. Perhaps the best demonstration that PAD-mediated citrullination can facilitate nonautoimmune or microbialinduced inflammatory events is the recent finding that PAD activity is strongly upregulated in inflamed tissue following a sterile skin punch biopsy procedure in mice [79]. Thus, it can be inferred that PAD-mediated citrullination plays a critical and fundamental role in inflammatory events induced by a range of pathologies, both infectious and noninfectious.

7.3. Role of PADs in Chemokine Signaling. PAD2 and PAD4 are highly expressed in peripheral blood mononuclear cells such as NK cells, T cells, B cells, and monocytes [16] and, thus, are likely to be the main PAD "players" in chemokine signaling. Chemokines are important for the proper recruitment of leukocytes to the site of inflammation. The chemokine-receptor system can be dramatically modified in neoplasms, especially at the invasive edges, and can act as a proangiogenic and a prodesmoplastic mediator. Several chemokines, including CXCL1, 2, and 8 have been found to exert effects on tumor cell growth. The CXC group of chemokines, with the tripeptide (GluLeu-Arg/ELR) at the amino-terminus of the CXC motif $(\mathrm{ELR}+)$, is proangiogenic and stimulates cell migration and proliferation. The capacity of chemokines to activate or repress biological pathways depends, in part, upon posttranslational modifications such as glycosylation and on proteolytic processing of the chemokine's $\mathrm{N}$ - or Cterminus [80]. Importantly, PADs have recently been found to citrullinate CXCL5, CXCL8, CCL17, and CCL26 [81], thus directly modulating the inflammatory milieu. Furthermore, chemokine citrullination does not appear to be a rare event in vivo, as, for example, CXCL8 was found to be citrullinated at arginine 5 in $14 \%$ of all blood leukocyte derived CXCL8 [81]. While the role of citrullinated chemokines in the inflammatory process is currently coming to light, their effect in cancer progression has yet to be investigated. However, given the strong links between inflammation and cancer progression, these observations support the hypothesis that PAD activity may play an important role in regulating the inflammatory milieu of the cancer microenvironment.

\section{Conclusions}

Protein citrullination is emerging as a critical posttranslational modification in developmental biology, inflammation, and cancer pathogenesis. With respect to cancer, PAD enzymes are now being identified as important potential players in tumor progression that both regulate transcriptional activity and modulate the inflammatory microenvironment via cytokine citrullination. The major likely roles of PADs in cancer pathogenesis are summarized in Figure 7. Given these emerging links between PADs and cancer biology, a better understanding of the upstream mechanisms that induce PAD expression, and the downstream mechanisms by which PADs regulate gene expression and inflammatory events will likely advance our understanding of tumor biology. Furthermore, the upregulation of specific PAD isozymes and activity at critical points of tumor progression raises the possibility that these enzymes, and their resulting 
posttranslational modifications, can function as novel cancer biomarkers. Lastly, the observations that (1) PAD inhibitors reduce inflammatory symptoms in mouse models of disease, (2) the link between PADs, inflammation, and cancer is currently unfolding, and (3) next generation isozymespecific PAD inhibitors are currently being developed, raise the possibility that the use of PAD inhibitors in preclinical and clinical cancer therapies may soon be realized.

\section{Acknowledgments}

This work was supported by (1) DOD Era of Hope Scholar Award (Grant W871XWH-07-1-0372) to S.A.C., and (2) NIGMS Grant RO1 GM079357 to P.R.T. The authors would like to thank Lynne Anguish for providing technical assistance in microscopic imaging and Kelly Emmott for helping with the xenograft studies.

\section{References}

[1] E. R. Vossenaar, A. J. W. Zendman, W. J. Van Venrooij, and G. J. M. Pruijn, "PAD, a growing family of citrullinating enzymes: genes, features and involvement in disease," BioEssays, vol. 25, no. 11, pp. 1106-1118, 2003.

[2] R. B. Denman, "PAD: the smoking gun behind arginine methylation signaling?" BioEssays, vol. 27, no. 3, pp. 242-246, 2005.

[3] C. Anzilotti, F. Pratesi, C. Tommasi, and P. Migliorini, "Peptidylarginine deiminase 4 and citrullination in health and disease," Autoimmunity Reviews, vol. 9, no. 3, pp. 158-160, 2010.

[4] X. Chang, R. Yamada, A. Suzuki et al., "Localization of peptidylarginine deiminase 4 (PADI4) and citrullinated protein in synovial tissue of rheumatoid arthritis," Rheumatology, vol. 44, no. 1, pp. 40-50, 2005.

[5] X. Chang and J. Han, "Expression of peptidylarginine deiminase type 4 (PAD4) in various tumors," Molecular Carcinogenesis, vol. 45, no. 3, pp. 183-196, 2006.

[6] J. E. Jones, C. P. Causey, B. Knuckley, J. L. Slack-Noyes, and P. R. Thompson, "Protein arginine deiminase 4 (PAD4): current understanding and future therapeutic potential," Current Opinion in Drug Discovery and Development, vol. 12, no. 5, pp. 616-627, 2009.

[7] M. De Ceuleneer, K. Van Steendam, M. Dhaenens, and D. Deforce, "In vivo relevance of citrullinated proteins and the challenges in their detection," Proteomics, vol. 12, pp. 752-760, 2012.

[8] S. Chavanas, M. C. Méchin, H. Takahara et al., "Comparative analysis of the mouse and human peptidylarginine deiminase gene clusters reveals highly conserved non-coding segments and a new human gene, PADI6," Gene, vol. 330, no. 1-2, pp. 19-27, 2004.

[9] E. R. Vossenaar, S. Nijenhuis, M. M. A. Helsen et al., "Citrullination of synovial proteins in murine models of rheumatoid arthritis," Arthritis and Rheumatism, vol. 48, no. 9, pp. 2489-2500, 2003.

[10] R. E. Ellsworth, A. Vertrees, B. Love et al., "Chromosomal alterations associated with the transition from in situ to invasive breast cancer," Annals of Surgical Oncology, vol. 15, no. 9, pp. 2519-2525, 2008.

[11] E. Darrah, A. Rosen, J. T. Giles, and F. Andrade, "Peptidylarginine deiminase 2, 3 and 4 have distinct specificities against cellular substrates: novel insights into autoantigen selection in rheumatoid arthritis," Annals of the Rheumatic Diseases, vol. 71, pp. 92-98, 2012.

[12] B. Knuckley, C. P. Causey, J. E. Jones et al., "Substrate specificity and kinetic studies of PADs 1, 3, and 4 identify potent and selective inhibitors of protein arginine deiminase 3," Biochemistry, vol. 49, no. 23, pp. 4852-4863, 2010.

[13] L. B. Pritzker, S. Joshi, G. Harauz, and M. A. Moscarello, "Deimination of myelin basic protein. 2. Effect of methylation of MBP on its deimination by peptidylarginine deiminase," Biochemistry, vol. 39, no. 18, pp. 5382-5388, 2000.

[14] L. B. Pritzker, S. Joshi, J. J. Gowan, G. Harauz, and M. A. Moscarello, "Deimination of myelin basic protein. 1. Effect of deimination of arginyl residues of myelin basic protein on its structure and susceptibility to digestion by cathepsin D," Biochemistry, vol. 39, no. 18, pp. 5374-5381, 2000.

[15] A. A. Musse, L. Zhen, C. A. Ackerley et al., "Peptidylarginine deiminase 2 (PAD2) overexpression in transgenic mice leads to myelin loss in the central nervous system," Disease Models and Mechanisms, vol. 1, no. 4-5, pp. 229-240, 2008.

[16] E. R. Vossenaar, T. R. D. Radstake, A. Van Der Heijden et al., "Expression and activity of citrullinating peptidylarginine deiminase enzymes in monocytes and macrophages," Annals of the Rheumatic Diseases, vol. 63, no. 4, pp. 373-381, 2004.

[17] B. D. Cherrington, E. Morency, A. M. Struble, S. A. Coonrod, and J. J. Wakshlag, "Potential role for peptidylarginine deiminase 2 (PAD2) in citrullination of canine mammary epithelial cell histones," PLoS ONE, vol. 5, no. 7, Article ID e11768, 2010.

[18] B. D. Cherrington, X. Zhang, J. L. McElwee, E. Morency, L. J. Anguish, and S. A. Coonrod, "Potential role for PAD2 in gene regulation in breast cancer cells," PloS ONE, vol. 7, Article ID e41242, 2012.

[19] X. Zhang, M. Bolt, M. J. Guertin et al., "Peptidylarginine deiminase 2-catalyzed histone $\mathrm{H} 3$ arginine 26 citrullination facilitates estrogen receptor $\alpha$ target gene activation," Proceedings of the National Academy of Sciences of the United States of America, vol. 109, no. 33, pp. 13331-13336, 2012.

[20] R. Nachat, M. C. Méchin, H. Takahara et al., "Peptidylarginine deiminase isoforms 1-3 are expressed in the epidermis and involved in the deimination of K1 and filaggrin," Journal of Investigative Dermatology, vol. 124, no. 2, pp. 384-393, 2005.

[21] X. Chang and K. Fang, "PADI4 and tumourigenesis," Cancer Cell International, vol. 10, article 7, 2010.

[22] K. Nakashima, T. Hagiwara, and M. Yamada, "Nuclear localization of peptidylarginine deiminase $\mathrm{V}$ and histone deimination in granulocytes," Journal of Biological Chemistry, vol. 277, no. 51, pp. 49562-49568, 2002.

[23] X. Chang, R. Yamada, T. Sawada, A. Suzuki, Y. Kochi, and K. Yamamoto, "The inhibition of antithrombin by peptidylarginine deiminase 4 may contribute to pathogenesis of rheumatoid arthritis," Rheumatology, vol. 44, no. 3, pp. 293298, 2005.

[24] A. Ordóñez, I. Martínez-Martínez, F. J. Corrales et al., "Effect of citrullination on the function and conformation of antithrombin," The FEBS Journal, vol. 276, no. 22, pp. 67636772, 2009.

[25] A. Ordóñez, J. Yélamos, S. Pedersen et al., "Increased levels of citrullinated antithrombin in plasma of patients with rheumatoid arthritis and colorectal adenocarcinoma determined by a newly developed ELISA using a specific monoclonal antibody," Thrombosis and Haemostasis, vol. 104, no. 6, pp. 1143-1149, 2010. 
[26] C. Tanikawa, K. Ueda, H. Nakagawa, N. Yoshida, Y. Nakamura, and K. Matsuda, "Regulation of protein citrullination through p53/PADI4 Network in DNA damage response," Cancer Research, vol. 69, no. 22, pp. 8761-8769, 2009.

[27] Q. Guo and W. Fast, "Citrullination of Inhibitor of Growth 4 (ING4) by Peptidylarginine Deminase 4 (PAD4) disrupts the interaction between ING4 and p53," Journal of Biological Chemistry, vol. 286, no. 19, pp. 17069-17078, 2011.

[28] H. Yao, P. Li, B. J. Venters et al., "Histone Arg modifications and p53 regulate the expression of OKL38, a mediator of apoptosis," Journal of Biological Chemistry, vol. 283, no. 29, pp. 20060-20068, 2008.

[29] P. Li, H. Yao, Z. Zhang et al., "Regulation of p53 target gene expression by peptidylarginine deiminase 4," Molecular and Cellular Biology, vol. 28, no. 15, pp. 4745-4758, 2008.

[30] X. Zhang, M. J. Gamble, S. Stadler et al., "Genome-Wide analysis reveals PADI4 cooperates with Elk-1 to activate C-Fos expression in breast cancer cells," PLoS Genetics, vol. 7, no. 6, Article ID e1002112, 2011.

[31] Y. Wang, M. Li, S. Stadler et al., "Histone hypercitrullination mediates chromatin decondensation and neutrophil extracellular trap formation," Journal of Cell Biology, vol. 184, no. 2, pp. 205-213, 2009.

[32] C. Tanikawa, M. Espinosa, A. Suzuki et al., "Regulation of histone modification and chromatin structure by the p53PADI4 pathway," Nature Communications, vol. 3, article 676, 2012.

[33] H. L. Rust and P. R. Thompson, "Kinase consensus sequences: a breeding ground for crosstalk," ACS Chemical Biology, vol. 6, pp. 881-892, 2011.

[34] P. Li, D. Wang, H. Yao et al., "Coordination of PAD4 and HDAC2 in the regulation of p53-target gene expression," Oncogene, vol. 29, no. 21, pp. 3153-3162, 2010.

[35] P. Yurttas, A. M. Vitale, R. J. Fitzhenry et al., "Role for PADI6 and the cytoplasmic lattices in ribosomal storage in oocytes and translational control in the early mouse embryo," Development, vol. 135, no. 15, pp. 2627-2636, 2008.

[36] S. N. Stacey, D. F. Gudbjartsson, P. Sulem et al., "Common variants on 1 p36 and 1q42 are associated with cutaneous basal cell carcinoma but not with melanoma or pigmentation traits," Nature Genetics, vol. 40, no. 11, pp. 1313-1318, 2008.

[37] P. Yurttas, E. Morency, and S. A. Coonrod, "Use of proteomics to identify highly abundant maternal factors that drive the egg-to-embryo transition," Reproduction, vol. 139, no. 5, pp. 809-823, 2010.

[38] L. Li, P. Zheng, and J. Dean, "Maternal control of early mouse development," Development, vol. 137, no. 6, pp. 859-870, 2010.

[39] T. Senshu, K. Akiyama, S. Nagata, K. Watanabe, and K. Hikichi, "Peptidylarginine deiminase in rat pituitary: sex difference, estrous cycle-related changes, and estrogen dependence," Endocrinology, vol. 124, no. 6, pp. 2666-2670, 1989.

[40] H. Takahara, M. Tsuchida, M. Kusubata, K. Akutsu, and S. K. Tagami Sugawara, "Peptidylarginine deiminase of the mouse. Distribution, properties, and immunocytochemical localization," Journal of Biological Chemistry, vol. 264, no. 22, pp. 13361-13368, 1989.

[41] K. Watanabe, M. Nomoto, S. Nagata et al., "The rat peptidylarginine deiminase-encoding gene: structural analysis and the 5'-flanking sequence," Gene, vol. 114, no. 2, pp. 261-265, 1992.

[42] S. Nagata, T. Uehara, K. Inoue, and T. Senshu, "Increased peptidylarginine deiminase expression during induction of prolactin biosynthesis in a growth-hormone-producing rat pituitary cell line, MtT/S," Journal of Cellular Physiology, vol. 150, no. 2, pp. 426-432, 1992.

[43] T. Barrett, D. B. Troup, S. E. Wilhite et al., "NCBI GEO: archive for high-throughput functional genomic data," Nucleic Acids Research, vol. 37, no. 1, pp. D885-D890, 2009.

[44] S. C. Hewitt, B. J. Deroo, K. Hansen et al., "Estrogen receptor-dependent genomic responses in the uterus mirror the biphasic physiological response to estrogen," Molecular Endocrinology, vol. 17, no. 10, pp. 2070-2083, 2003.

[45] H. Takahara, M. Kusubata, M. Tsuchida, T. Kohsaka, S. Tagami, and K. Sugawara, "Expression of peptidylarginine deiminase in the uterine epithelial cells of mouse is dependent on estrogen," Journal of Biological Chemistry, vol. 267, no. 1, pp. 520-525, 1992.

[46] S. Horibata, S. A. Coonrod, and B. D. Cherrington, "Role for peptidylarginine deiminase enzymes in disease and female reproduction," The Journal of Reproduction and Development, vol. 58, pp. 274-282, 2012.

[47] S. Dong, Z. Zhang, and H. Takahara, "Estrogen-enhanced peptidylarginine deiminase type IV gene (PADI4) expression in MCF-7 cells is mediated by estrogen receptor- $\alpha$-promoted transfactors activator protein-1, nuclear factor-Y, and Sp1," Molecular Endocrinology, vol. 21, no. 7, pp. 1617-1629, 2007.

[48] Y. Wang, J. Wysocka, J. Sayegh et al., "Human PAD4 regulates histone arginine methylation levels via demethylimination," Science, vol. 306, no. 5694, pp. 279-283, 2004.

[49] A. A. Chumanevich, C. P. Causey, B. A. Knuckley et al., "Suppression of colitis in mice by Cl-amidine: a novel peptidylarginine deiminase inhibitor," American Journal of Physiology, vol. 300, no. 6, pp. G929-G938, 2011.

[50] L. R. Tait, R. J. Pauley, S. J. Santner et al., "Dynamic stromal-epithelial interactions during progression of MCF10DCIS.com xenografts," International Journal of Cancer, vol. 120, no. 10, pp. 2127-2134, 2007.

[51] F. R. Miller, S. J. Santner, L. Tait, and P. J. Dawson, "MCF10DCIS.com xenograft model of human comedo ductal carcinoma in situ," Journal of the National Cancer Institute, vol. 92, no. 14, pp. 1185-1186, 2000.

[52] L. Wang, X. Chang, G. Yuan, Y. Zhao, and P. Wang, "Expression of peptidylarginine deiminase type 4 in ovarian tumors," International Journal of Biological Sciences, vol. 6, no. 5, pp. 454-464, 2010.

[53] X. Chang, J. Han, L. Pang, Y. Zhao, Y. Yang, and Z. Shen, "Increased PADI4 expression in blood and tissues of patients with malignant tumors," BMC Cancer, vol. 9, article 40, 2009.

[54] B. D. Cherrington, S. Mohanan, A. N. Diep et al., "Comparative analysis of peptidylarginine deiminase-2 expression in canine, feline and human mammary tumours," Journal of Comparative Pathology, vol. 147, pp. 139-146, 2012.

[55] J. L. Slack, C. P. Causey, and P. R. Thompson, "Protein arginine deiminase 4: a target for an epigenetic cancer therapy," Cellular and Molecular Life Sciences, vol. 68, no. 4, pp. 709-720, 2011.

[56] Y. Luo, K. Arita, M. Bhatia et al., "Inhibitors and inactivators of protein arginine deiminase 4: functional and structural characterization," Biochemistry, vol. 45, no. 39, pp. 1172711736, 2006.

[57] Y. Luo, B. Knuckley, Y. H. Lee, M. R. Stallcup, and P. R. Thompson, "A fluoroacetamidine-based inactivator of protein arginine deiminase 4: design, synthesis, and in vitro and in vivo evaluation," Journal of the American Chemical Society, vol. 128, no. 4, pp. 1092-1093, 2006.

[58] B. Knuckley, Y. Luo, and P. R. Thompson, "Profiling Protein Arginine Deiminase 4 (PAD4): a novel screen to identify PAD4 
inhibitors," Bioorganic and Medicinal Chemistry, vol. 16, no. 2, pp. 739-745, 2008.

[59] Y. Wang, P. Li, S. Wang et al., "Anticancer PAD inhibitors regulate the autophagy flux and the mammalian target of rapamycin complex 1 activity," The Journal of Biological Chemistry, vol. 287, pp. 25941-25953, 2012.

[60] C. W. Van, M. G. Alison, K. B. Nirmal et al., "N- $\alpha$-benzoyl-N5(2-chloro-1-iminoethyl)-L-ornithine amide, a protein arginine deiminase inhibitor, reduces the severity of murine collagen-induced arthritis," Journal of Immunology, vol. 186, no. 7, pp. 4396-4404, 2011.

[61] B. L. Pierce, M. L. Neuhouser, M. H. Wener et al., "Correlates of circulating C-reactive protein and serum amyloid A concentrations in breast cancer survivors," Breast Cancer Research and Treatment, vol. 114, no. 1, pp. 155-167, 2009.

[62] O. I. Ahmed, A. M. Adel, D. R. Diab, and N. S. Gobran, "Prognostic value of serum level of interleukin- 6 and interleukin- 8 in metastatic breast cancer patients," The Egyptian Journal of Immunology, vol. 13, no. 2, pp. 61-68, 2006.

[63] T. Bachelot, I. Ray-Coquard, C. Menetrier-Caux, M. Rastkha, A. Duc, and J. Y. Blay, "Prognostic value of serum levels of interleukin 6 and of serum and plasma levels of vascular endothelial growth factor in hormone-refractory metastatic breast cancer patients," British Journal of Cancer, vol. 88, no. 11, pp. 1721-1726, 2003.

[64] S. W. Cole, "Chronic inflammation and breast cancer recurrence," Journal of Clinical Oncology, vol. 27, no. 21, pp. 3418 3419, 2009.

[65] R. Salgado, S. Junius, I. Benoy et al., "Circulating interleukin6 predicts survival in patients with metastatic breast cancer," International Journal of Cancer, vol. 103, no. 5, pp. 642-646, 2003.

[66] G. J. Zhang and I. Adachi, "Serum interleukin-6 levels correlate to tumor progression and prognosis in metastatic breast carcinoma," Anticancer Research, vol. 19, no. 2, pp. 1427-1432, 1999.

[67] L. M. Coussens and Z. Werb, "Inflammation and cancer," Nature, vol. 420, no. 6917, pp. 860-867, 2002.

[68] I. J. Fidler, "The pathogenesis of cancer metastasis: the 'seed and soil' hypothesis revisited," Nature Reviews Cancer, vol. 3, no. 6, pp. 453-458, 2003.

[69] I. Auger, C. Charpin, N. Balandraud, M. Martin, and J. Roudier, "Autoantibodies to PAD4 and BRAF in rheumatoid arthritis," Autoimmunity Reviews, vol. 11, no. 11, pp. 801-803, 2012.

[70] M. Saffarzadeh, C. Juenemann, M. A. Queisser et al., "Neutrophil extracellular traps directly induce epithelial and endothelial cell death: a predominant role of histones," PloS ONE, vol. 7, Article ID e32366, 2012.

[71] C. L. Liu, S. Tangsombatvisit, J. M. Rosenberg et al., "Specific post-translational histone modifications of neutrophil extracellular traps as immunogens and potential targets of lupus autoantibodies," Arthritis Research \& Therapy, vol. 14, article R25, 2012.

[72] B. Amulic and G. Hayes, "Neutrophil extracellular traps," Current Biology, vol. 21, no. 9, pp. R297-R298, 2011.

[73] P. Li, M. Li, M. R. Lindberg, M. J. Kennett, N. Xiong, and Y. Wang, "PAD4 is essential for antibacterial innate immunity mediated by neutrophil extracellular traps," Journal of Experimental Medicine, vol. 207, no. 9, pp. 1853-1862, 2010.

[74] L. Fan, D. He, Q. Wang et al., "Citrullinated vimentin stimulates proliferation, pro-inflammatory cytokine secretion, and PADI4 and RANKL expression of fibroblast-like synoviocytes in rheumatoid arthritis," Scandinavian Journal of Rheumatology. In press.

[75] H. J. Lee, M. Joo, R. Abdolrasulnia et al., "Peptidylarginine deiminase 2 suppresses inhibitory $\kappa \mathrm{B}$ kinase activity in lipopolysaccharide-stimulated RAW 264.7 macrophages," Journal of Biological Chemistry, vol. 285, no. 51, pp. 3965539662, 2010.

[76] V. Ruiz-Esquide, M. J. Gomara, V. I. Peinado et al., "Anticitrullinated peptide antibodies in the serum of heavy smokers without rheumatoid arthritis. A differential effect of chronic obstructive pulmonary disease?" Clinical Rheumatology, vol. 31, pp. 1047-1050, 2012.

[77] O. Kilsgard, P. Andersson, M. Malmsten et al., "Peptidylarginine deiminases present in the airways during tobacco smoking and inflammation can citrullinate the host defense peptide LL-37, resulting in altered activities," American Journal of Respiratory Cell and Molecular Biology, vol. 46, pp. 240-248, 2012.

[78] D. Makrygiannakis, E. Af Klint, I. E. Lundberg et al., "Citrullination is an inflammation-dependent process," Annals of the Rheumatic Diseases, vol. 65, no. 9, pp. 1219-1222, 2006.

[79] F. Coudane, M. C. Mechin, A. Huchenq et al., "Deimination and expression of peptidylarginine deiminases during cutaneous wound healing in mice," European Journal of Dermatology, vol. 21, no. 3, pp. 376-384, 2011.

[80] A. Mortier, M. Gouwy, J. Van Damme, and P. Proost, "Effect of posttranslational processing on the in vitro and in vivo activity of chemokines," Experimental Cell Research, vol. 317, no. 5, pp. 642-654, 2011.

[81] P. Proost, T. Loos, A. Mortier et al., "Citrullination of CXCL8 by peptidylarginine deiminase alters receptor usage, prevents proteolysis, and dampens tissue inflammation," Journal of Experimental Medicine, vol. 205, no. 9, pp. 2085-2097, 2008. 

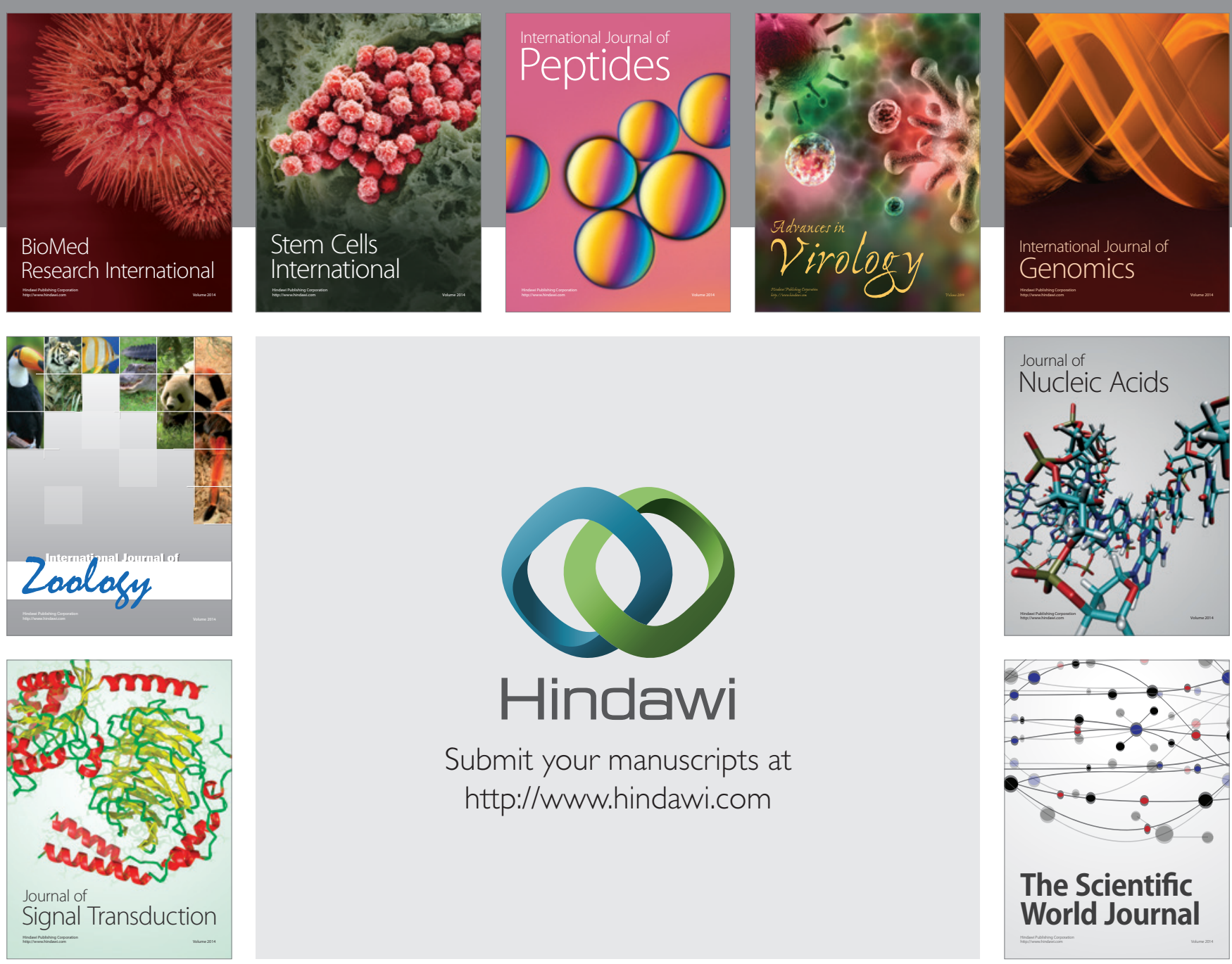

Submit your manuscripts at

http://www.hindawi.com
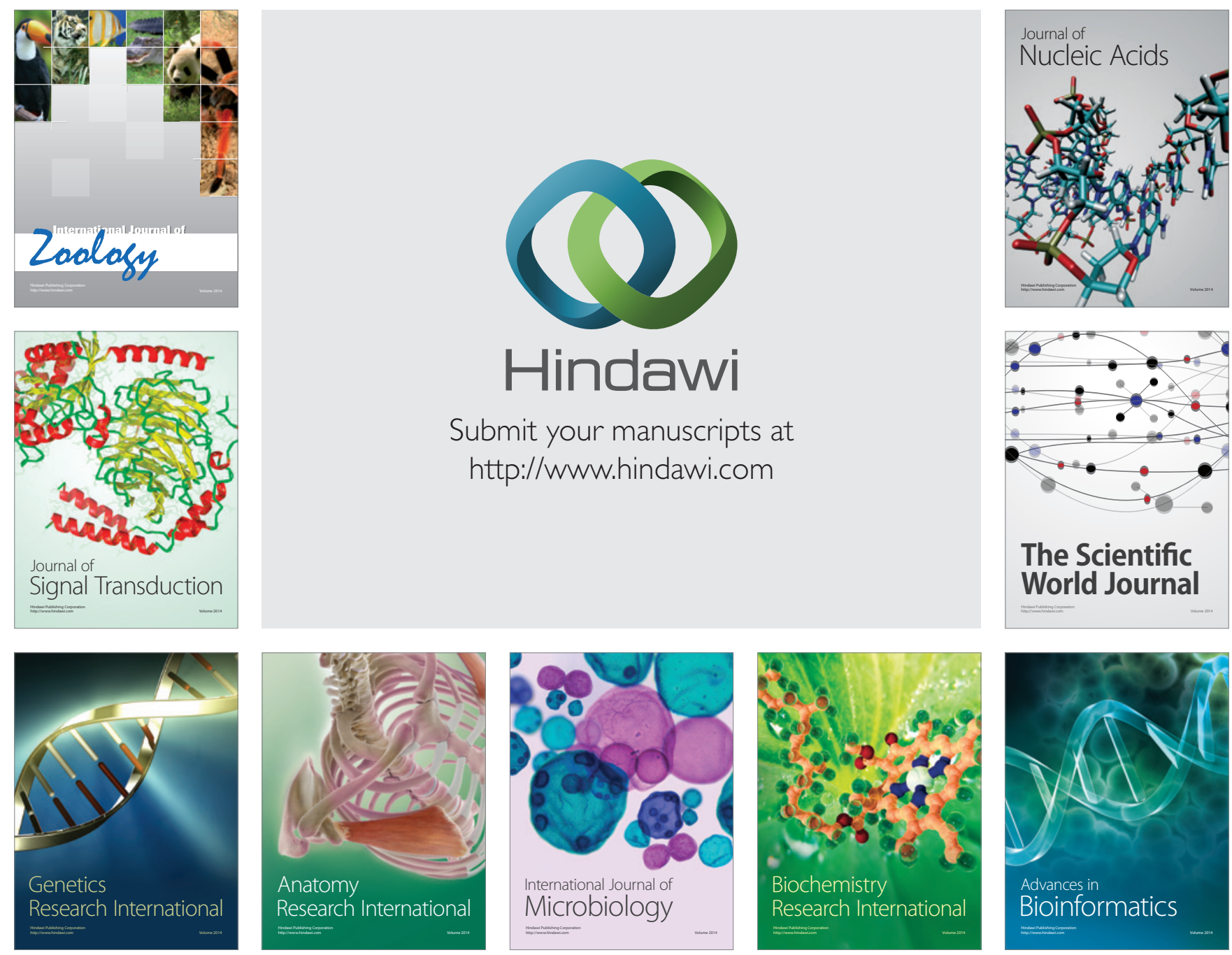

The Scientific World Journal
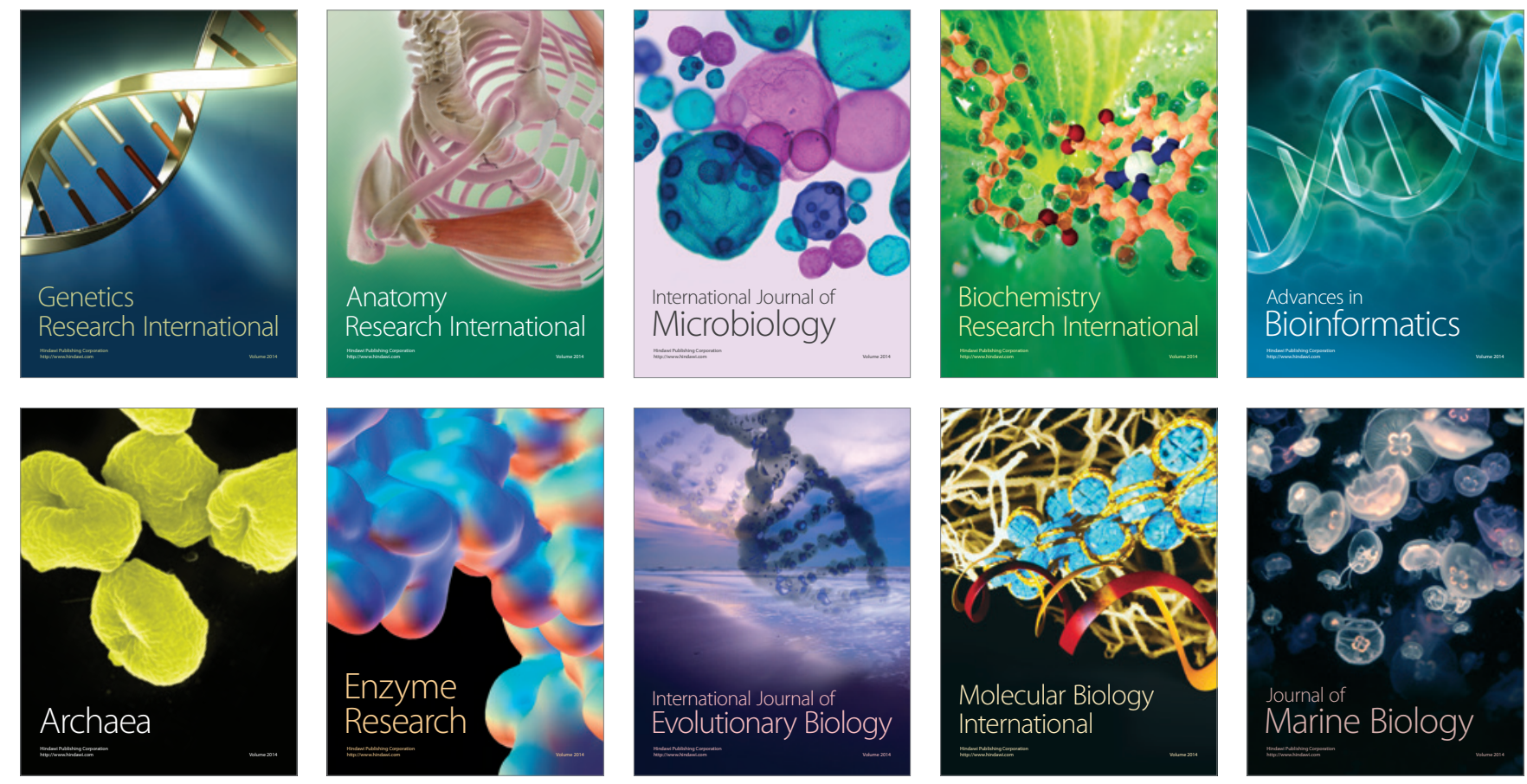